\title{
Influence of Different Training and Nutrition Levels on Growth and Yield of Tomato (Solanum lycopersicum L.) under Protected Condition
}

\author{
S.K. Anand*, N. Basavaraja, C.N. Hanchinamani, H.P. Hadimani, \\ I.B. Biradar and D. Satish
}

Department of Vegetable Science, Kittur Rani Channamma College of Horticulture, Arabhavi- 591 218, University of Horticultural Sciences, Bagalkot, Karnataka, India

*Corresponding author

\section{A B S T R A C T}

\begin{tabular}{|l|}
\hline Ke y w or d s \\
Tomato, Training, \\
Nutrition, Growth, \\
Yield, Economics \\
\hline Article Info \\
\hline Accepted: \\
24 August 2018 \\
Available Online: \\
10 September 2018 \\
\hline
\end{tabular}

Study on influence of training and nutrition on yield of tomato (Solanum lycopersicum L.) under protected condition was taken up at HRES, Hidakal Dam (near KRCCH, Arabhavi), Karnataka, during rabi 2017-2018. Experiment was laid out in two Factorial CRD Design, with three levels of training and pruning treatments and eight levels of nutrition having total 24 treatments, replicated thrice. Among 24 different treatment combinations of training and nutrition levels, the highest number of fruits per plant (57.42), yield per plot $(61.61 \mathrm{~kg} / \mathrm{ha})$, yield per hectare $(184.83 \mathrm{t} / \mathrm{ha})$ found highest in $\mathrm{T}_{2} \mathrm{~N}_{6}$ [Two stem $+75 \%$ $\mathrm{RDF}+$ Protein hydrolysates $(1.5 \mathrm{~g} / \mathrm{litre})+\mathrm{PSB}(2 \mathrm{~kg} / \mathrm{ha})+\mathrm{VAM}(2 \mathrm{~kg} / \mathrm{ha})]$ treatment. The highest plant height $\left(173.0 \mathrm{~cm}\right.$ ) found in $\mathrm{T}_{1} \mathrm{~N}_{6}$ (Single stem $+75 \% \mathrm{RDF}+$ Protein hydrolysates $(1.5 \mathrm{~g} /$ litre $)+$ PSB $(2 \mathrm{~kg} / \mathrm{ha})+$ VAM $(2 \mathrm{~kg} / \mathrm{ha})$. The highest plant spread $(\mathrm{N}-$ $\mathrm{S})$ and $(\mathrm{E}-\mathrm{W})(125.13 \mathrm{~cm})$ and $(117.79 \mathrm{~cm})$ and ascorbic acid $(15.93 \mathrm{mg})$ found in $\mathrm{T}_{0} \mathrm{~N}_{6}$ $[$ Control + 75\% RDF + Protein hydrolysates (1.5 g/litre) + PSB (2 kg/ha) + VAM (2 $\mathrm{kg} / \mathrm{ha})]$.

\section{Introduction}

Tomato (Solanum lycopersicum L.) is a popular vegetable crop grown throughout the world due to its wider adaptability. It belongs to family solanaceae having chromosome number $2 n=24$. Though the centre of origin is Peru Ecuador region, it was introduced to India in early $16^{\text {th }}$ century by eastern countries.

It is an annual and short lived perennial, herbaceous, typical day neutral and selfpollinated plant but certain percentage of cross pollination also occurs $(0.5$ to $4 \%)$. Crop is a warm season one, resistant to heat and drought and it grows under wide range of soil and climatic situation. It is considered as 'protective food' due to its nutritive value, antioxidant molecules like carotenoids (ascorbic acid, lycopene, vitamin E and phenol compounds, particularly flavonoids) (Septa $e t$ al., 2013). The presence of lycopene reduces the risk of heart attacks and many types of cancer (Dorgan et al., 1998; Clinton, 2005).

Protected cultivation of vegetables emphasizes the need for having appropriate plant densities 
and training systems in order to boost up the production by utilizing the available space and nutrient applied. Manipulation of plant architecture through training with appropriate spatial arrangements has also been revealed as a key management factor for getting maximum yield from greenhouse crops (Guo et al., 1991).

Thus there is a prime need to increase the productivity and production of tomato in the country and the state as well. Among the various factors responsible for low production are improper cultural operations and nutrient management are the important factors. Tomato yield could be increased substantially through improved production techniques like training and pruning Mantur and Patil (2008). However, the benefits of pruning according to Preece and Read (2005) include; while pruning improves the qualitative and quantitative characteristics of tomato fruits by limiting the vegetative growth and also allows more sunlight penetration. The training system emphasizes on the plant ability to obtain sunlight for growth. It is also important to maintain sufficient air circulation around the plants to reduce risks of pests. The leaves of a well-trained plant dry of faster, so bacterial and fungal pathogen have less opportunity to spread. Trained tomato plants are much easier to harvest and less damage is done to the vines. Hesami et al., (2012) found that trained and pruned tomato plants yielded higher than untrained and unpruned plants. The main reason for staking and supporting tomato plants is to keep plants and fruits off the ground. This reduces losses from fruit rots when fruit touch the soil land from sunburn when fruits are not shaded by foliage. Supported plants are easier to spray or dust for insect and disease control and easier to harvest than those sprawling on the ground. Beside staking, the another important factor among the various cultural practices for improving yield and quality in tomato is nutritional management of the crop. A bearing tomato plant removes considerable amount of nutrients from the soil. Therefore, balanced manuring and fertilization practices in vogue are generally empirical. Due to escalating cost of chemical fertilizers and objective of minimizing environmental pollution, the search of alternative source of plant nutrients is imperative. The integrated plant nutrient supply envisages conductive use of inorganic and organic sources of plant nutrients for crop productivity besides sustaining soil health.

There is a meagre work on training and nutrition in tomato. Therefore, there is a need for improvement and standardization of training and pruning with respect to nutrition combination in tomato genotype for yield and quality under protected condition especially for fresh market with high nutritive value and higher yield.

\section{Materials and Methods}

The experiment was conducted during rabi season of 2017-18 in protected condition at Horticultural Research and Extension Station, Hidkal Dam (Badakundri) near Belgaum, Kittur Rani Channamma College of Horticulture, Arabhavi, University of Horticultural Sciences, Bagalkot, Karnataka, India. The details of experimental techniques, materials used and methods followed during the experimentation have been described here under.

Training and pruning treatments viz., $\mathrm{T}_{0}$ : Control, $\mathrm{T}_{1}$ : Single stem, $\mathrm{T}_{2}$ : Double stem and Nutrition treatments viz., $\mathrm{N}_{0}$ : Recommended dose of fertilizer NPK @ 115:100:60 kg/ha + FYM (25 t/ha), $\quad \mathrm{N}_{1}: \quad 75 \% \quad \mathrm{RDF}+$ Vermicompost (1 t/ha), $\mathrm{N}_{2}: 50 \% \mathrm{RDF}+$ Vermicompost (2 t/ha), $\mathrm{N}_{3}: 25 \% \mathrm{RDF}+$ Vermicompost $(0.5 \mathrm{t} / \mathrm{ha})+$ Neem cake $(50$ $\mathrm{kg} / \mathrm{ha}), \mathrm{N}_{4}: 75 \% \mathrm{RDF}+\mathrm{PSB}(2 \mathrm{~kg} / \mathrm{ha})+$ VAM (2 kg/ha), $\mathrm{N}_{5}: 50 \% \mathrm{RDF}+\mathrm{PSB}$ (3 
$\mathrm{kg} / \mathrm{ha})+\mathrm{VAM}(3 \mathrm{~kg} / \mathrm{ha}), \mathrm{N}_{6}: 75 \% \mathrm{RDF}+$ Protein hydrolysates (1.5 g/litre) + PSB (2 $\mathrm{kg} / \mathrm{ha})+\mathrm{VAM}(2 \mathrm{~kg} / \mathrm{ha}), \mathrm{N}_{7}: 50 \% \mathrm{RDF}+$ Protein hydrolysates (1.5 g/litre) + PSB (3 $\mathrm{kg} / \mathrm{ha})+$ VAM $(3 \mathrm{~kg} / \mathrm{ha})$ with three replications. Thirty days old, good, vigorous and uniform height seedlings were selected and used for transplanting in the main experimental plots at a spacing of $60 \times 45 \mathrm{~cm}$ as per the treatment.

Water soluble chemical fertilizers were applied in split doses at weekly intervals over a period of 4 months starting from 15 days after transplanting through drip fertigation system.

For application of $P S B$ and $V A M$ culture in each bed as per treatment combination were applied in soil just before the transplanting of tomato seedlings followed by irrigation (Sharma, 2009).

A plant biostimulant Protein hydrolysates (PHs) were sprayed after the pruning of the plant.

Protein hydrolysates (PHs) are an important group of plant biostimulants based on a mixture of peptides and amino acids that have received increasing attention in the recent years due to their positive effects on crop performances (Colla et al., 2015).

Mulching was done to reduce weed problem in the beds. Only the space between the beds was kept weed free by manual weeding.

\section{Results and Discussion}

In the present investigations, attempts were made to study the influence of different training and nutrition levels on growth and yield of tomato (Solanum lycopersicum L.) under protected condition and the discussion of the results are as under.
Growth parameters i.e., plant height was highest in $\mathrm{T}_{1} \mathrm{~N}_{6}$ [Single stem $+75 \% \mathrm{RDF}+$ Protein hydrolysates (1.5 g/litre) + PSB (2 $\mathrm{kg} / \mathrm{ha})+\operatorname{VAM}(2 \mathrm{~kg} / \mathrm{ha})](173.00 \mathrm{~cm})$ and plant spread (N-S \& E-W) was highest in $\mathrm{T}_{0} \mathrm{~N}_{6}$ [Control $+75 \% \mathrm{RDF}+$ Protein hydrolysates $(1.5 \mathrm{~g} / \mathrm{litre})+$ PSB $(2 \mathrm{~kg} / \mathrm{ha})+$ VAM (2 $\mathrm{kg} / \mathrm{ha})](125.13 \mathrm{~cm} \& 117.79 \mathrm{~cm})$. Increase in plant height is due to the pinching of axillary shoots at their emergence and ultimately diverting the flow of nutrients and manufactured food material towards apical growing point and greater exposure of plants to light leading to higher cell multiplication and photosynthetic activity. The congenial microclimate (optimum carbon dioxide, temperature and relative humidity) prevailed inside the polyhouse might have favoured the increased growth rate of plants. Similar results were obtained by Nimje et al., (1990) in sweet pepper and brinjal. The availability of more growth space where in plants were able to exploit more nutrients from the soil and light sources. These results are in corroborate with the findings of Ara et al., (2007), Muhammad and Singh (2014) and Yadav (2017) in tomato. Protein hydrolysates have been identified to improve the performance of several horticultural crops, including increased shoot, and root biomass and productivity. Application of protein hydrolysates to plant leaves and roots has been shown to increase $\mathrm{Fe}$ and $\mathrm{N}$ metabolism, nutrient uptake and water and nutrient use efficiencies for both macro and microelements (Colla et al., 2015). The similar beneficial effect of combined use of fertilizers, organic manure and biofertilizers on plant height was also recorded by Kumaran et al., (1998) in tomato. The significant role of VAM fungi in increasing the plant height of tomato was also reported earlier by Gosavi (2010) and Yeptho et al., (2010) in tomato.

Plant spread was higher in control (No pruning) it may be due to not pinching the axillary shoots at their emergence and 
ultimately there will be increase in the canopy of the plant. These results also corroborate with the findings of Muhammad and Singh (2014) and Yadav (2017) in tomato. The improvement in the plant spread at all stages of growth with application of 75 per cent RDF along with bio-fertilizer might be due to better uptake and translocation of nitrogen to the growing plants as a result of their quick availability in the treatment. Nitrogen makes up part of the chlorophyll in plants. Light energy is taken by the chlorophyll and used to make sugars for the plant. It helps the nutrients and water be taken up into the plant (Xin and Jun, 2014). The similar beneficial effect of combined use of fertilizers, organic manure and biofertilizers on plant height was recorded by Kumaran et al., (1998) in tomato. Root colonization by VAM fungi enhances plant growth. This is due to improved nutrient uptake and increased protection against soil borne pathogenic fungi (Bidartondo et al., 2002). The significant role of VAM fungi in increasing the plant spread of tomato was reported earlier by Yeptho et al., (2010) in tomato.

The fruit yield (yield per plant, yield per plot, yield per hectare) and yield attribute such as fruits per plant were significantly higher in $\mathrm{T}_{2} \mathrm{~N}_{6}$ [Two stem $+75 \% \mathrm{RDF}+$ Protein hydrolysates (1.5 g/litre) + PSB (2 kg/ha) + VAM (2 kg/ha)] (6.16 kg, $61.61 \mathrm{~kg} / \mathrm{plot}$, $184.83 \mathrm{t} / \mathrm{ha}$ and 57.42 respectively) treatment followed by $\mathrm{T}_{2} \mathrm{~N}_{7}$ [Two stem $+50 \% \mathrm{RDF}+$ Protein hydrolysates (1.5 g/litre) + PSB (3 $\mathrm{kg} / \mathrm{ha})+$ VAM (3 kg/ha)] (5.88 kg, 58.84 $\mathrm{kg} / \mathrm{plot}, 176.51 \mathrm{t} / \mathrm{ha}$ and 55.42 respectively) treatment, whereas average fruit weight were higher in treatment $\mathrm{T}_{1} \mathrm{~N}_{6}$ [Single stem $+75 \%$ $\mathrm{RDF}+$ Protein hydrolysates (1.5 g/litre) + PSB (2 kg/ha) + VAM (2 kg/ha)] (116.13 g), whereas fruit yield and yield attributes were lower in $\mathrm{T}_{0} \mathrm{~N}_{3}$ [Control $+25 \% \mathrm{RDF}+$ Vermicompost $(0.5 \mathrm{t} / \mathrm{ha})+$ Neem cake $(50$ $\mathrm{kg} / \mathrm{ha}$ )] followed by control $\mathrm{T}_{0} \mathrm{~N}_{0}$ (Control +
Recommended dose of fertilizer NPK @ $115: 100: 60 \mathrm{~kg} / \mathrm{ha}$ ). Increase in fruit yield is due to more number of fruits and average fruit weight. The two stem training system noticed significant and maximum fruit yield per hectare over other levels of training systems. This is attributed to the presence of higher number of fruits per plant, average fruit weight resulting into highest fruit yield per plant and ultimately the yield per plot under two stem systems. The increase in yield of tomato per plant, per plot and per hectare under naturally ventilated polyhouse was attributed to the favourable climatic conditions like optimum temperature, light intensity and relative humidity that prevailed and resulted in higher vegetative growth, contributing to more number of flowers, more number of fruits, higher percentage of fruit set, maximum fruit weight and fruit volume.

Similar findings of increased yields under medium cost polyhouse was also reported by Basavaraja et al., (2003) in capsicum.The results of present findings are in agreement with the findings of Khoshkam et al., (2014) and Razzak et al., (2013) in tomato and Lal et al., (2014) in capsicum who expressed number of fruits per plant is the key factor in obtaining higher yield. Several scientists have reported fruit length, fruit diameter had paramount effect due to due to complementary action of phosphorous and potassium which helps in synthesis of auxins which are responsible for the cell elongation by increasing the cell permeability to water and osmotic solutes of the cells.

Besides, increase in the fruit size might be due to the higher uptake of nutrients and more food material synthesis by plant when treated with bio-fertilizers. FYM is store houses of the nutrients in soil, which enhance the fruit length, fruit diameter, fruit weight and fruit volume Mohankumar and Narasegowda (2010) (Table 1-5). 
Table.1 Effect of different training and nutrition levels on plant height $(\mathrm{cm})$ in tomato under protected condition

\begin{tabular}{|c|c|c|c|c|}
\hline Treatments & 30 DAT & $60 \mathrm{DAT}$ & 90 DAT & 120 DAT \\
\hline $\mathbf{T}_{0}$ & 57.99 & 117.8 & 127.60 & 139.68 \\
\hline$T_{1}$ & 58.15 & 122.14 & 150.67 & 170.68 \\
\hline$T_{2}$ & 58.07 & 119.25 & 135.66 & 161.48 \\
\hline SEm \pm & 0.376 & 0.496 & 0.190 & 0.143 \\
\hline CD at $5 \%$ & NS & 1.489 & 0.570 & 0.431 \\
\hline $\mathbf{N}_{0}$ & 56.01 & 118.06 & 135.60 & 154.43 \\
\hline $\mathbf{N}_{1}$ & 56.55 & 119.92 & 137.47 & 156.00 \\
\hline $\mathbf{N}_{2}$ & 56.57 & 118.43 & 136.47 & 157.67 \\
\hline $\mathbf{N}_{3}$ & 54.58 & 117.94 & 135.64 & 153.57 \\
\hline$\overline{N_{4}}$ & 60.22 & 120.79 & 139.20 & 157.67 \\
\hline $\mathbf{N}_{5}$ & 60.07 & 120.59 & 138.30 & 159.10 \\
\hline$\overline{N_{6}}$ & 60.53 & 121.22 & 141.13 & 160.12 \\
\hline $\mathbf{N}_{7}$ & 60.04 & 120.96 & 140.05 & 159.57 \\
\hline SEm \pm & 0.615 & 0.810 & 0.310 & 0.234 \\
\hline CD at $5 \%$ & 1.845 & 2.432 & 0.931 & 0.703 \\
\hline \multicolumn{5}{|c|}{ Interaction effects } \\
\hline $\mathbf{T}_{0} \mathbf{N}_{0}$ & 55.95 & 116.72 & 126.80 & 136.70 \\
\hline $\mathbf{T}_{0} \mathbf{N}_{1}$ & 57.07 & 119.52 & 127.80 & 139.40 \\
\hline $\mathrm{T}_{0} \mathbf{N}_{2}$ & 56.32 & 117.93 & 127.40 & 139.80 \\
\hline $\mathbf{T}_{0} \mathbf{N}_{3}$ & 54.60 & 115.37 & 126.13 & 135.50 \\
\hline $\mathbf{T}_{0} \mathbf{N}_{4}$ & 60.51 & 117.62 & 128.24 & 139.00 \\
\hline $\mathbf{T}_{0} \mathbf{N}_{5}$ & 60.09 & 117.22 & 127.37 & 141.60 \\
\hline $\mathrm{T}_{0} \mathrm{~N}_{6}$ & 60.23 & 119.12 & 128.24 & 143.00 \\
\hline $\mathrm{T}_{0} \mathrm{~N}_{7}$ & 59.12 & 119.02 & 128.30 & 142.40 \\
\hline $\mathrm{T}_{1} \mathbf{N}_{0}$ & 56.10 & 119.72 & 148.40 & 168.40 \\
\hline $\mathbf{T}_{1} \mathbf{N}_{1}$ & 56.28 & 121.22 & 150.50 & 168.80 \\
\hline $\mathrm{T}_{1} \mathbf{N}_{2}$ & 57.05 & 118.63 & 149.50 & 171.40 \\
\hline $\mathrm{T}_{1} \mathbf{N}_{3}$ & 54.51 & 121.89 & 149.80 & 172.40 \\
\hline $\mathbf{T}_{1} \mathbf{N}_{4}$ & 60.05 & 123.72 & 151.57 & 173.00 \\
\hline $\mathbf{T}_{1} \mathbf{N}_{5}$ & 60.09 & 123.85 & 150.93 & 172.60 \\
\hline $\mathrm{T}_{1} \mathbf{N}_{6}$ & 60.65 & 124.22 & 153.00 & 173.00 \\
\hline $\mathbf{T}_{1} \mathbf{N}_{7}$ & 60.50 & 124.02 & 151.76 & 172.60 \\
\hline $\mathbf{T}_{2} \mathbf{N}_{0}$ & 55.90 & 117.72 & 131.60 & 158.20 \\
\hline $\mathrm{T}_{2} \mathrm{~N}_{1}$ & 56.30 & 119.02 & 134.10 & 159.80 \\
\hline $\mathbf{T}_{2} \mathbf{N}_{2}$ & 56.35 & 118.72 & 132.60 & 162.20 \\
\hline $\mathrm{T}_{2} \mathbf{N}_{3}$ & 54.62 & 116.57 & 131.00 & 157.40 \\
\hline $\mathrm{T}_{2} \mathbf{N}_{4}$ & 60.10 & 121.02 & 137.80 & 162.60 \\
\hline $\mathrm{T}_{2} \mathbf{N}_{5}$ & 60.05 & 120.82 & 136.60 & 163.30 \\
\hline $\mathrm{T}_{2} \mathrm{~N}_{6}$ & 60.70 & 120.95 & 141.50 & 164.60 \\
\hline $\mathbf{T}_{2} \mathbf{N}_{7}$ & 60.49 & 120.83 & 140.10 & 163.70 \\
\hline SEm \pm & 1.065 & 1.404 & 0.537 & 0.406 \\
\hline CD at $5 \%$ & NS & NS & 1.613 & 1.219 \\
\hline
\end{tabular}

\section{$\mathrm{T}_{0}$ : Control (No pruning), $\mathrm{T}_{1}$ : Single stem, $\mathrm{T}_{2}$ : Two stem}

$\mathrm{N}_{0}$ : RDF NPK @ 115:100:60 kg/ha.

$\mathrm{N}_{2}: 50 \%$ RDF + Vermicompost (2t/ha)

$\mathrm{N}_{4}: 75 \% \mathrm{RDF}+$ PSB (2kg/ha) +VAM (2kg/ha),

$\mathrm{N}_{6}: \mathbf{7 5} \%$ RDF + Protein hydrolysates $(1.5$

g/litre) + PSB (2kg/ha) + VAM (2kg/ha)
$\mathrm{N}_{1}: 75 \% \mathrm{RDF}+$ Vermicompost $(1 \mathrm{t} / \mathrm{ha})$

$\mathrm{N}_{3}: 25 \% \mathrm{RDF}+$ Vermicompost $(0.5 \mathrm{t} / \mathrm{ha})+$ Neem cake $(50 \mathrm{~kg} / \mathrm{ha})$

$\mathrm{N}_{5}: 50 \% \mathrm{RDF}+\mathrm{PSB}(3 \mathrm{~kg} / \mathrm{ha})+\mathrm{VAM}(3 \mathrm{~kg} / \mathrm{ha})$

$\mathrm{N}_{7}: 50 \% \mathrm{RDF}+$ Protein hydrolysates (1.5 g/litre) + PSB $(3 \mathrm{~kg} / \mathrm{ha})$ + VAM (3kg/ha) 
Table.2 Effect of different training and nutrition levels on plant spread $(\mathrm{cm})(\mathrm{N}-\mathrm{S})$ in tomato under protected condition

\begin{tabular}{|c|c|c|c|c|}
\hline Treatments & $30 \mathrm{DAT}$ & $60 \mathrm{DAT}$ & 90 DAT & $120 \mathrm{DAT}$ \\
\hline $\mathbf{T}_{0}$ & 51.97 & 87.21 & 102.10 & 121.10 \\
\hline $\mathbf{T}_{1}$ & 51.54 & 66.87 & 85.58 & 95.88 \\
\hline $\mathbf{T}_{2}$ & 50.82 & 72.85 & 93.14 & 111.21 \\
\hline SEm \pm & 0.393 & 0.007 & 0.128 & 0.128 \\
\hline $\mathrm{CD}$ at $5 \%$ & NS & 0.021 & 0.365 & 0.365 \\
\hline $\mathbf{N}_{0}$ & 46.90 & 71.43 & 90.53 & 106.90 \\
\hline $\mathrm{N}_{1}$ & 49.97 & 75.13 & 93.00 & 109.07 \\
\hline $\mathbf{N}_{2}$ & 48.79 & 73.37 & 91.90 & 107.94 \\
\hline $\mathbf{N}_{3}$ & 46.67 & 68.40 & 88.60 & 104.61 \\
\hline $\mathbf{N}_{4}$ & 53.80 & 78.38 & 95.04 & 111.87 \\
\hline $\mathbf{N}_{5}$ & 52.44 & 76.67 & 93.42 & 106.86 \\
\hline $\mathbf{N}_{6}$ & 57.24 & 81.50 & 96.67 & 114.82 \\
\hline $\mathbf{N}_{7}$ & 55.72 & 80.26 & 95.55 & 113.08 \\
\hline SEm \pm & 0.641 & 0.012 & 0.209 & 0.200 \\
\hline CD at $5 \%$ & 1.825 & 0.035 & 0.596 & 0.585 \\
\hline \multicolumn{5}{|c|}{ Interaction effects } \\
\hline $\mathrm{T}_{0} \mathbf{N}_{0}$ & 48.37 & 82.96 & 100.33 & 119.33 \\
\hline $\mathbf{T}_{0} \mathbf{N}_{1}$ & 49.57 & 86.96 & 102.13 & 121.13 \\
\hline $\mathbf{T}_{0} \mathbf{N}_{2}$ & 48.97 & 84.46 & 100.33 & 119.33 \\
\hline $\mathrm{T}_{0} \mathbf{N}_{\mathbf{3}}$ & 48.27 & 79.76 & 97.53 & 116.53 \\
\hline $\mathrm{T}_{0} \mathrm{~N}_{4}$ & 54.77 & 90.16 & 104.33 & 123.33 \\
\hline $\mathrm{T}_{0} \mathbf{N}_{5}$ & 52.97 & 88.66 & 101.83 & 120.83 \\
\hline $\mathrm{T}_{0} \mathrm{~N}_{6}$ & 57.07 & 93.17 & 106.13 & 125.13 \\
\hline $\mathrm{T}_{0} \mathrm{~N}_{7}$ & 55.77 & 91.54 & 104.13 & 123.13 \\
\hline $\mathbf{T}_{1} \mathbf{N}_{0}$ & 46.77 & 63.16 & 81.93 & 92.53 \\
\hline $\mathbf{T}_{1} \mathbf{N}_{1}$ & 51.17 & 66.16 & 84.53 & 94.43 \\
\hline $\mathbf{T}_{1} \mathbf{N}_{2}$ & 49.57 & 64.66 & 84.53 & 93.98 \\
\hline $\mathbf{T}_{1} \mathbf{N}_{3}$ & 46.57 & 59.17 & 80.93 & 90.35 \\
\hline $\mathbf{T}_{1} \mathbf{N}_{4}$ & 53.80 & 69.17 & 85.33 & 97.68 \\
\hline$\overline{\mathbf{T}_{1} \mathbf{N}_{5}}$ & 51.77 & 67.28 & 84.33 & 96.45 \\
\hline $\mathrm{T}_{1} \mathbf{N}_{6}$ & 56.89 & 73.16 & 85.33 & 101.98 \\
\hline $\mathbf{T}_{1} \mathbf{N}_{7}$ & 55.78 & 72.17 & 85.33 & 99.58 \\
\hline $\mathrm{T}_{2} \mathbf{N}_{0}$ & 45.57 & 68.16 & 89.33 & 108.83 \\
\hline $\mathbf{T}_{2} \mathbf{N}_{1}$ & 49.17 & 72.26 & 92.33 & 111.65 \\
\hline $\mathbf{T}_{2} \mathbf{N}_{2}$ & 47.83 & 70.98 & 90.83 & 110.49 \\
\hline $\mathbf{T}_{2} \mathbf{N}_{3}$ & 45.17 & 66.26 & 87.33 & 106.93 \\
\hline $\mathbf{T}_{2} \mathbf{N}_{4}$ & 52.82 & 75.81 & 95.45 & 114.59 \\
\hline $\mathrm{T}_{2} \mathbf{N}_{5}$ & 52.57 & 74.06 & 94.08 & 103.28 \\
\hline $\mathrm{T}_{2} \mathbf{N}_{6}$ & 57.77 & 78.17 & 98.53 & 117.33 \\
\hline $\mathrm{T}_{2} \mathbf{N}_{7}$ & 55.62 & 77.06 & 97.18 & 116.53 \\
\hline SEm \pm & 1.111 & 0.021 & 0.363 & 0.343 \\
\hline CD at $5 \%$ & NS & 0.061 & 1.032 & 1.022 \\
\hline
\end{tabular}

\section{$\mathrm{T}_{0}$ : Control (No pruning), $\mathrm{T}_{1}$ : Single stem, $\mathrm{T}_{2}$ : Two stem}

$\mathrm{N}_{0}$ : RDF NPK @ 115:100:60 kg/ha.

$\mathrm{N}_{2}: 50 \%$ RDF + Vermicompost (2t/ha)

$\mathrm{N}_{4}: 75 \%$ RDF+ PSB (2kg/ha) +VAM (2kg/ha),

$\mathrm{N}_{6}: \mathbf{7 5} \%$ RDF + Protein hydrolysates (1.5

g/litre) + PSB (2kg/ha) + VAM (2kg/ha)
$\mathrm{N}_{1}: 75 \% \mathrm{RDF}+$ Vermicompost $(1 \mathrm{t} / \mathrm{ha})$

$\mathrm{N}_{3}: 25 \% \mathrm{RDF}+$ Vermicompost $(0.5 \mathrm{t} / \mathrm{ha})+$ Neem cake $(50 \mathrm{~kg} / \mathrm{ha})$

$\mathrm{N}_{5}: 50 \% \mathrm{RDF}+\mathrm{PSB}(3 \mathrm{~kg} / \mathrm{ha})+\mathrm{VAM}(3 \mathrm{~kg} / \mathrm{ha})$

$\mathrm{N}_{7}: 50 \% \mathrm{RDF}+$ Protein hydrolysates $(1.5 \mathrm{~g} / \mathrm{litre})+\mathrm{PSB}(3 \mathrm{~kg} / \mathrm{ha})$

+ VAM (3kg/ha) 
Table.3 Effect of different training and nutrition levels on plant spread $(\mathrm{cm})(\mathrm{E}-\mathrm{W})$ in tomato under protected condition

\begin{tabular}{|c|c|c|c|c|}
\hline Treatments & 30 DAT & $60 \mathrm{DAT}$ & 90 DAT & $120 \mathrm{DAT}$ \\
\hline $\mathrm{T}_{0}$ & 48.09 & 75.45 & 93.92 & 111.64 \\
\hline $\mathrm{T}_{1}$ & 47.36 & 63.75 & 76.95 & 85.88 \\
\hline $\mathbf{T}_{2}$ & 48.36 & 70.74 & 85.88 & 95.81 \\
\hline SEm \pm & 0.584 & 0.128 & 0.077 & 0.077 \\
\hline CD at $5 \%$ & NS & 0.365 & 0.221 & 0.221 \\
\hline $\mathbf{N}_{0}$ & 45.18 & 66.20 & 81.71 & 94.53 \\
\hline $\mathbf{N}_{1}$ & 47.31 & 69.00 & 85.04 & 97.48 \\
\hline $\mathbf{N}_{2}$ & 46.60 & 67.62 & 83.58 & 96.16 \\
\hline $\mathbf{N}_{3}$ & 44.25 & 64.24 & 79.32 & 89.23 \\
\hline $\mathbf{N}_{4}$ & 49.44 & 72.74 & 87.89 & 100.45 \\
\hline $\mathbf{N}_{5}$ & 48.42 & 71.29 & 86.46 & 98.98 \\
\hline $\mathbf{N}_{6}$ & 52.96 & 75.22 & 91.02 & 103.27 \\
\hline $\mathbf{N}_{7}$ & 51.99 & 73.55 & 89.62 & 102.10 \\
\hline SEm \pm & 0.954 & 0.209 & 0.127 & 0.116 \\
\hline CD at $5 \%$ & 2.714 & 0.596 & 0.361 & 0.350 \\
\hline \multicolumn{5}{|c|}{ Interaction effects } \\
\hline $\mathrm{T}_{0} \mathrm{~N}_{0}$ & 45 & 72.33 & 90.17 & 109.67 \\
\hline $\mathrm{T}_{0} \mathrm{~N}_{1}$ & 47.2 & 74.33 & 93.17 & 112.25 \\
\hline $\mathrm{T}_{0} \mathrm{~N}_{2}$ & 46.75 & 73.58 & 91.77 & 110.82 \\
\hline $\mathbf{T}_{0} \mathbf{N}_{3}$ & 44.25 & 70.83 & 87.97 & 97.80 \\
\hline $\mathbf{T}_{0} \mathbf{N}_{4}$ & 49.53 & 77.83 & 95.99 & 114.88 \\
\hline $\mathrm{T}_{0} \mathrm{~N}_{5}$ & 48.23 & 76.56 & 94.91 & 113.17 \\
\hline $\mathrm{T}_{0} \mathrm{~N}_{6}$ & 52.56 & 79.58 & 99.52 & 117.79 \\
\hline $\mathrm{T}_{0} \mathrm{~N}_{7}$ & 51.23 & 78.54 & 97.88 & 116.77 \\
\hline $\mathbf{T}_{1} \mathbf{N}_{0}$ & 44.2 & 59.53 & 73.17 & 82.17 \\
\hline $\mathrm{T}_{1} \mathrm{~N}_{1}$ & 46.23 & 62.53 & 76.07 & 85.12 \\
\hline $\mathrm{T}_{1} \mathbf{N}_{2}$ & 45.75 & 60.93 & 74.99 & 83.88 \\
\hline $\mathrm{T}_{1} \mathrm{~N}_{3}$ & 43.3 & 57.43 & 70.23 & 79.82 \\
\hline $\mathbf{T}_{1} \mathbf{N}_{4}$ & 48.23 & 66.73 & 79.38 & 88.32 \\
\hline $\mathrm{T}_{1} \mathrm{~N}_{5}$ & 47.51 & 65.65 & 77.60 & 87.07 \\
\hline$T_{1} N_{6}$ & 52.2 & 69.54 & 82.93 & 91.02 \\
\hline $\mathbf{T}_{1} \mathbf{N}_{7}$ & 51.43 & 67.64 & 81.23 & 89.65 \\
\hline $\mathbf{T}_{2} \mathbf{N}_{0}$ & 46.34 & 66.73 & 81.79 & 91.77 \\
\hline $\mathbf{T}_{2} \mathbf{N}_{1}$ & 48.5 & 70.13 & 85.90 & 95.08 \\
\hline $\mathbf{T}_{2} \mathbf{N}_{2}$ & 47.3 & 68.33 & 83.99 & 93.79 \\
\hline $\mathbf{T}_{2} \mathbf{N}_{3}$ & 45.21 & 64.45 & 79.77 & 90.08 \\
\hline $\mathbf{T}_{2} \mathbf{N}_{4}$ & 50.56 & 73.65 & 88.32 & 98.17 \\
\hline $\mathbf{T}_{2} \mathbf{N}_{5}$ & 49.53 & 71.64 & 86.88 & 96.70 \\
\hline $\mathbf{T}_{2} \mathbf{N}_{6}$ & 54.12 & 76.54 & 90.62 & 101.02 \\
\hline $\mathbf{T}_{2} \mathbf{N}_{7}$ & 53.31 & 74.45 & 89.77 & 99.88 \\
\hline SEm \pm & 1.653 & 0.363 & 0.220 & 0.210 \\
\hline $\mathrm{CD}$ at $5 \%$ & NS & 1.032 & 0.626 & 0.615 \\
\hline
\end{tabular}

\section{$\mathrm{T}_{0}$ : Control (No pruning), $\mathrm{T}_{1}$ : Single stem, $\mathrm{T}_{2}$ : Two stem}

$\mathrm{N}_{0}$ : RDF NPK @ 115:100:60 kg/ha.

$\mathrm{N}_{2}: 50 \%$ RDF + Vermicompost (2t/ha)

$\mathrm{N}_{4}:$ 75\% RDF+ PSB (2kg/ha) +VAM (2kg/ha),

$\mathrm{N}_{6}: 75 \%$ RDF + Protein hydrolysates (1.5 g/litre $)+$ PSB (2kg/ha) + VAM (2kg/ha)
$\mathrm{N}_{1}: 75 \% \mathrm{RDF}+$ Vermicompost (1t/ha)

$\mathrm{N}_{3}: 25 \% \mathrm{RDF}+$ Vermicompost $(0.5 \mathrm{t} / \mathrm{ha})+$ Neem cake $(50 \mathrm{~kg} / \mathrm{ha})$

$\mathrm{N}_{5}: 50 \% \mathrm{RDF}+\mathrm{PSB}(3 \mathrm{~kg} / \mathrm{ha})+\mathrm{VAM}(3 \mathrm{~kg} / \mathrm{ha})$

$\mathrm{N}_{7}: 50 \% \mathrm{RDF}+$ Protein hydrolysates $(1.5 \mathrm{~g} /$ litre $)+$ $\operatorname{PSB}(3 \mathrm{~kg} / \mathrm{ha})+\mathrm{VAM}(3 \mathrm{~kg} / \mathrm{ha})$ 
Table.4 Effect of different training and nutrition levels on yield and yield attributing parameters in tomato under protected condition

\begin{tabular}{|c|c|c|c|c|c|}
\hline Treatments & $\begin{array}{c}\text { Number of fruits } \\
\text { per plant }\end{array}$ & $\begin{array}{l}\text { Average fruit } \\
\text { weight (g) }\end{array}$ & $\begin{array}{l}\text { Yield per } \\
\text { plant (kg) }\end{array}$ & $\begin{array}{l}\text { Yield per } \\
\text { plot }(\mathbf{k g})\end{array}$ & $\begin{array}{c}\text { Yield per } \\
\text { hectare (t/ha) }\end{array}$ \\
\hline$T_{0}$ & 35.74 & 83.96 & 3.01 & 30.10 & 90.30 \\
\hline$T_{1}$ & 28.84 & 112.22 & 3.25 & 32.51 & 97.52 \\
\hline$T_{2}$ & 47.94 & 103.08 & 4.96 & 49.63 & 148.88 \\
\hline SEm & 0.37 & 0.046 & 0.035 & 0.347 & 1.042 \\
\hline CD at $5 \%$ & 1.101 & 0.175 & 0.104 & 1.042 & 3.125 \\
\hline $\mathbf{N}_{0}$ & 31.82 & 96.7 & 3.05 & 30.46 & 91.38 \\
\hline $\mathbf{N}_{1}$ & 36.25 & 99.16 & 3.57 & 35.75 & 107.24 \\
\hline $\mathbf{N}_{2}$ & 34.75 & 98.14 & 3.38 & 33.85 & 101.55 \\
\hline $\mathbf{N}_{3}$ & 28.25 & 95.63 & 2.67 & 26.66 & 79.99 \\
\hline $\mathbf{N}_{4}$ & 41.08 & 101.74 & 4.18 & 41.83 & 125.48 \\
\hline$N_{5}$ & 39.42 & 100.21 & 3.95 & 39.48 & 118.44 \\
\hline $\mathbf{N}_{6}$ & 45.42 & 103.66 & 4.70 & 47.01 & 141.03 \\
\hline $\mathbf{N}_{7}$ & 43.08 & 102.79 & 4.43 & 44.25 & 132.76 \\
\hline SEm \pm & 0.599 & 0.095 & 0.057 & 0.567 & 1.701 \\
\hline CD at $5 \%$ & 1.797 & 0.286 & 0.170 & 1.701 & 5.103 \\
\hline \multicolumn{6}{|c|}{ Interaction effects } \\
\hline $\mathbf{T}_{0} \mathbf{N}_{0}$ & 32.02 & 81.27 & 2.60 & 26.02 & 78.05 \\
\hline $\mathbf{T}_{0} \mathbf{N}_{1}$ & 34.92 & 83.3 & 2.91 & 29.08 & 87.25 \\
\hline $\mathbf{T}_{0} \mathbf{N}_{2}$ & 34.42 & 82.76 & 2.85 & 28.48 & 85.44 \\
\hline $\mathbf{T}_{0} \mathbf{N}_{3}$ & 28.92 & 80.12 & 2.32 & 23.16 & 69.49 \\
\hline $\mathbf{T}_{0} \mathbf{N}_{4}$ & 37.42 & 85.80 & 3.21 & 32.10 & 96.30 \\
\hline $\mathbf{T}_{0} \mathbf{N}_{5}$ & 36.42 & 83.99 & 3.06 & 30.59 & 91.76 \\
\hline$T_{0} N_{6}$ & 42.42 & 87.54 & 3.71 & 37.13 & 111.39 \\
\hline $\mathbf{T}_{0} \mathbf{N}_{7}$ & 39.42 & 86.88 & 3.42 & 34.24 & 102.73 \\
\hline $\mathrm{T}_{1} \mathbf{N}_{0}$ & 22.82 & 109.28 & 2.49 & 24.93 & 74.80 \\
\hline$T_{1} N_{1}$ & 27.42 & 111.86 & 3.07 & 30.67 & 92.00 \\
\hline $\mathrm{T}_{1} \mathbf{N}_{2}$ & 25.42 & 110.24 & 2.80 & 28.02 & 84.05 \\
\hline$T_{1} \mathbf{N}_{3}$ & 19.42 & 108.65 & 2.11 & 21.09 & 63.28 \\
\hline $\mathbf{T}_{1} \mathbf{N}_{4}$ & 33.42 & 113.87 & 3.80 & 38.05 & 114.15 \\
\hline$T_{1} N_{5}$ & 31.42 & 112.44 & 3.53 & 35.32 & 105.97 \\
\hline$T_{1} N_{6}$ & 36.42 & 116.13 & 4.23 & 42.29 & 126.87 \\
\hline $\mathbf{T}_{1} \mathbf{N}_{7}$ & 34.42 & 115.31 & 3.97 & 39.68 & 119.05 \\
\hline $\mathrm{T}_{2} \mathrm{~N}_{0}$ & 40.62 & 99.54 & 4.04 & 40.43 & 121.28 \\
\hline $\mathbf{T}_{2} \mathbf{N}_{1}$ & 46.42 & 102.33 & 4.75 & 47.49 & 142.48 \\
\hline $\mathrm{T}_{2} \mathrm{~N}_{2}$ & 44.42 & 101.42 & 4.50 & 45.05 & 135.14 \\
\hline $\mathbf{T}_{2} \mathbf{N}_{3}$ & 36.42 & 98.12 & 3.57 & 35.73 & 107.19 \\
\hline $\mathbf{T}_{2} \mathbf{N}_{4}$ & 52.42 & 105.56 & 5.53 & 55.33 & 165.99 \\
\hline $\mathrm{T}_{2} \mathrm{~N}_{5}$ & 50.42 & 104.19 & 5.25 & 52.53 & 157.59 \\
\hline $\mathbf{T}_{2} \mathbf{N}_{6}$ & 57.42 & 107.3 & 6.16 & 61.61 & 184.83 \\
\hline $\mathrm{T}_{2} \mathrm{~N}_{7}$ & 55.42 & 106.17 & 5.88 & 58.84 & 176.51 \\
\hline SEm \pm & 1.038 & 0.165 & 0.098 & 0.982 & 2.946 \\
\hline CD at $5 \%$ & 3.113 & 0.496 & 0.295 & 2.946 & 8.838 \\
\hline \multicolumn{6}{|c|}{$T_{0}:$ Control (No pruning), $T_{1}:$ Single stem, $T_{2}:$ Two stem } \\
\hline \multirow{2}{*}{\multicolumn{3}{|c|}{$\begin{array}{l}\mathrm{N}_{0}: \text { RDF NPK @ 115:100:60 kg/ha. } \\
\mathrm{N}_{2}: 50 \% \text { RDF + Vermicompost (2t/ha) }\end{array}$}} & \multicolumn{3}{|c|}{$\mathrm{N}_{1}: 75 \% \mathrm{RDF}+$ Vermicompost (1t/ha) } \\
\hline & & & \multicolumn{3}{|c|}{$\begin{array}{l}\mathrm{N}_{3}: 25 \% \mathrm{RDF}+\text { Vermicompost }(0.5 \mathrm{t} / \mathrm{ha})+\text { Neem cake } \\
(50 \mathrm{~kg} / \mathrm{ha})\end{array}$} \\
\hline \multicolumn{3}{|c|}{$\mathrm{N}_{4}: 75 \%$ RDF+ PSB (2kg/ha) +VAM (2kg/ha), } & \multicolumn{3}{|c|}{$\mathrm{N}_{5}: 50 \% \mathrm{RDF}+\mathrm{PSB}(3 \mathrm{~kg} / \mathrm{ha})+\mathrm{VAM}(3 \mathrm{~kg} / \mathrm{ha})$} \\
\hline \multicolumn{3}{|c|}{$\begin{array}{l}\text { N6:75\% RDF + Protein hydrolysates (1.5 g/litre) + } \\
\text { PSB (2kg/ha) + VAM (2kg/ha) }\end{array}$} & \multicolumn{3}{|c|}{$\begin{array}{l}\mathrm{N}_{7}: 50 \% \mathrm{RDF}+\text { Protein hydrolysates }(1.5 \mathrm{~g} / \text { litre })+ \\
\text { PSB(3kg/ha) + VAM (3kg/ha) }\end{array}$} \\
\hline
\end{tabular}


Table.5 Benefit: Cost ratio of different treatments in training and nutrition levels in tomato crop under protected condition

\begin{tabular}{|c|c|c|c|c|}
\hline Treatment & $\begin{array}{l}\text { Total cost of } \\
\text { production } \\
\text { (₹) }\end{array}$ & $\begin{array}{l}\text { Gross returns } \\
\text { (₹) }\end{array}$ & $\begin{array}{l}\text { Net returns } \\
(₹)\end{array}$ & B:C Ratio \\
\hline $\mathbf{T}_{0} \mathbf{N}_{0}$ & 32,736 & 66,480 & 33,744 & 1.03 \\
\hline $\mathrm{T}_{0} \mathbf{N}_{1}$ & $32,908.5$ & 74,320 & $41,411.5$ & 1.25 \\
\hline $\mathbf{T}_{0} \mathbf{N}_{2}$ & 33,081 & 72,780 & 39,699 & 1.20 \\
\hline $\mathbf{T}_{0} \mathbf{N}_{3}$ & $32,703.5$ & 59,200 & $26,496.5$ & 0.81 \\
\hline $\mathrm{T}_{0} \mathbf{N}_{4}$ & $32,928.5$ & 82,040 & $49,111.5$ & 1.49 \\
\hline $\mathbf{T}_{0} \mathbf{N}_{5}$ & 33,161 & 78,160 & 44,999 & 1.35 \\
\hline $\mathbf{T}_{0} \mathbf{N}_{6}$ & $33,338.5$ & 94,900 & $61,561.5$ & 1.84 \\
\hline $\mathbf{T}_{0} \mathbf{N}_{7}$ & 33,371 & 87,520 & 54,149 & 1.62 \\
\hline $\mathbf{T}_{1} \mathbf{N}_{0}$ & 38,336 & 63,720 & 25,384 & 0.66 \\
\hline $\mathrm{T}_{1} \mathrm{~N}_{1}$ & $38,508.5$ & 78,380 & $39,871.5$ & 1.03 \\
\hline $\mathbf{T}_{1} \mathbf{N}_{2}$ & 38,241 & 71,600 & 33,359 & 0.87 \\
\hline $\mathbf{T}_{\mathbf{1}} \mathbf{N}_{\mathbf{3}}$ & $38,303.5$ & 53,900 & $15,596.5$ & 0.40 \\
\hline $\mathbf{T}_{\mathbf{1}} \mathbf{N}_{4}$ & $38,528.5$ & 97,240 & $58,711.5$ & 1.52 \\
\hline $\mathbf{T}_{1} \mathbf{N}_{5}$ & 38,561 & 90,280 & 51,719 & 1.34 \\
\hline $\mathbf{T}_{1} \mathbf{N}_{6}$ & $38,938.5$ & $1,08,080$ & $69,141.5$ & 1.77 \\
\hline $\mathbf{T}_{1} \mathbf{N}_{7}$ & $38,971.5$ & $1,01,420$ & $62,448.5$ & 1.60 \\
\hline $\mathbf{T}_{2} \mathbf{N}_{0}$ & 38,336 & $1,03,320$ & 64,984 & 1.69 \\
\hline $\mathrm{T}_{2} \mathrm{~N}_{1}$ & $38,508.5$ & $1,21,380$ & $82,871.5$ & 2.15 \\
\hline $\mathbf{T}_{2} \mathbf{N}_{2}$ & 38,681 & $1,15,120$ & 76,439 & 1.97 \\
\hline $\mathbf{T}_{2} \mathbf{N}_{3}$ & $38,303.5$ & 91,320 & $53,016.6$ & 1.38 \\
\hline $\mathbf{T}_{2} \mathbf{N}_{4}$ & 38,530 & $1,41,420$ & $1,02,890$ & 2.67 \\
\hline $\mathbf{T}_{2} \mathbf{N}_{5}$ & 38,561 & $1,34,260$ & 95,699 & 2.48 \\
\hline $\mathbf{T}_{2} \mathbf{N}_{6}$ & $38,938.5$ & $1,57,460$ & $1,18,521.5$ & 3.03 \\
\hline $\mathbf{T}_{2} \mathbf{N}_{7}$ & 38,971 & $1,50,380$ & $1,11,409$ & 2.85 \\
\hline
\end{tabular}

Potassium triggers activation of enzymes and is essential for production of Adenosine Triphosphate (ATP). VAM fungi act as root extensions that increase the volume of soil influenced and exploited by plants. VAM enlarges the root areas of host plants and improves its efficiency of water and nutrient absorption. PSB secrets a soluble greenish fluorescent pigment called fluorescein, particularly under conditions of low iron availability (Ganeshan and Kumar, 2005). There are many reports indicating increased fruit yield due to the application of organic manures along with inorganic fertilizers in accordance with the present investigation as also recorded by Manoj (2014) and Ranjith and Bandopadhyay (2014). Similarly, Koukounararas et al., (2013) who opined that the application (through roots or foliage) of a protein hydrolysates commercial product (Amino 16) could increase greenhouse tomato yield and yield components (fruit mean weight and number) under varying fertilisation doses. It is rather evident that the application of amino acid sand peptides in the nutrient solution has a beneficial effect on the 
leaf mineral status, in particular cations such as $\mathrm{K}, \mathrm{Ca}, \mathrm{Mg}, \mathrm{Fe}, \mathrm{Cu}$ and $\mathrm{Zn}$ (Garcia et al., 2011), consequently resulting in better growth of the crop.

The treatment combination $\mathrm{T}_{2} \mathrm{~N}_{6}$ [Two stem + $75 \% \mathrm{RDF}+$ Protein hydrolysates (1.5 g/litre) + PSB $(2 \mathrm{~kg} / \mathrm{ha})+$ VAM $(2 \mathrm{~kg} / \mathrm{ha})]$ is found to give highest benefit cost ratio (3.03) compared to lowest benefit cost ratio $(0.40)$. Because of increased growth and yield which fetches more price and will give higher net returns and benefit cost ratio. The poor growth and yield parameters leads to lowest benefit cost ratio $(0.40)$ in $\mathrm{T}_{1} \mathrm{~N}_{3}$ [Single stem $+25 \% \mathrm{RDF}+$ Vermicompost $(0.5 \mathrm{t} / \mathrm{ha})+$ Neem cake $(50 \mathrm{~kg} / \mathrm{ha})]$. Similar findings were also reported by Mohankumar and Narase Gowda (2010) who recorded maximum cost benefit ratio in tomato provided with integrated application of organic and inorganic inputs.

The study revealed that maximum total fruit yield is coupled with favourable growth characters could be achieved by training and nutrition. Hence, highest Benefit: Cost ratio (3.03) was recorded with treatment $\mathrm{T}_{2} \mathrm{~N}_{6}$ (Two stem $+75 \% \mathrm{RDF}+$ Protein hydrolysates (1.5 g/litre) + PSB (2 kg/ha) + VAM (2 kg/ha)) mainly due to total yield (184.83 t/ha).

\section{References}

Ara, N., Bashar, M. K., Begum, S. and Kakon, S. S., 2007, Effect of spacing and stem pruning on the growth and yield of tomato. International Journal of Sustainable Crop Production, 2(3): 35-39.

Basavaraja, N., Nandi, V. R. and Praveen, J., 2003, Protected cultivation of capsicum and bhendi. Proc. of All India seminar on potential and prospects for protective cultivation organized by the
Institute of Engineers, Ahmadnagar, December (12-13): 197-199.

Bidartondo, M. I., Redecker, D., Hijri, I., Wiemken, A., Bruns, T. D., Dominguez, L., Seesic, A., Leake, J. and Read, D. J, 2002, Epiparasitic plants specialized on arbuscular mycorrhizal fungi. Nature, 419: 389392.

Clinton, S. K., 2005, Tomatoes or lycopene: a role in prostate carcinogenesis. $J$. of Nutrition., 135(8): 2057-2059.

Colla, G., Nardi, S., Cardarelli, M., Ertani, A., Lucini, L., Canaguier, R. and Rouphael, Y., 2015, Protein hydrolysates as biostimulants in horticulture. Sci. Hortic., 1: 1-10.

Dorgan, J. F., Sowell, A., Swanson, C. A., Potischman, N., Miller, R., Schussler, N. and Stephenson, J. H., 1998, Relationship of serum carotenoids, retinol, alpha tocopherol and selenium with breast cancer risk: results from a prospective study in Columbia, Missouri (USA). Cancer Causes Control, 9(1): 89-97.

Ganeshan, G. and Kumar, M., 2005, Pseudomonas fluorescens, a potential bacterial antagonist to control plant diseases. Journal of Plant Interactions, 1(3): 123-134.

Garcia, A.L., Madrid, R., Gimeno, V., Rodriguez-Ortega, W.M., Nicolas, N., Garcia-Sanchez, F., 2011, The effects of amino acids fertilization incorporatedto the nutrient solution on mineral composition and growth in tomato seedlings. Span. J. Agric. Res. 9: 852861.

Gosavi, P. U., Kamble, A. B. and Pandure, B. S., 2010, Effect of organic manures and biofertilizers on quality of tomato fruits. Asian. J. Horti., 5(2): 376-378.

Guo, C. Y., Fujime, T., Hirose and Kato, T., 1990, Effects of the number of training shoots, raising period of seedlings and 
planting density on growth, fruiting and yield of sweet pepper. J. Japan Soc., Hortic. Sci., 59: 763-770.

Hesami, A., Khorami, S. S. and Hossein, S. S., 2012, Effect of shoot pruning and flower thinning on quality and quantity of semi-determinate tomato. Not. Sci. Bio., 4(1): 108-111.

Khoshkam, S., Seyedi, Z. and Ahmad, A., 2014, The impact of different plant training systems on quantitative and qualitative parameters of greenhouse tomato cultivars. International Journal of Farming and Allied Sciences, 3(6): 659-663.

Koukounararas, A., Tsouvaltzis, P., Siomos, A. S., 2013, Effect of root and foliar application of amino acids on the growth and yield of greenhouse tomato indifferent fertilization levels. J. Food Agric. Environ., 11: 644-648.

Kurnaran, S.S., Natrajan, S. and Thamburaj, S. 1998. Effect of organic and inorganic fertilizers on growth, yield and quality of tomato. South Indian Horticulture, 46(3/6): 203-205.

Lal, M., Kanwar, H. S. and Kanwar, R., 2014, Impact of spacing and training on seed yield of capsicum, (Capsicum annuum L.) under protected conditions. Int. J. Farm Sci, 4(3): 42-48.

Manoj, K., 2014, Impact of front line demonstration of INM on yield in tomato. Hort flora Res. Spectrum., 3(3): 295-297.

Mantur, S. M. and Patil, S. R., 2008, Influence of spacing and pruning on yield of tomato grown under shade house. Karnataka J. Agric. Sci., 21(1): 97-98.

Mohankumar, A. B. and Narasegowda, N. C., 2010, Effect of different organic manure and inorganic fertilizers on growth and yield of brinjal (Solanum melongena L.). The Asian J. of Hort., 5(2): 444 449 .
Muhammad, A. and Singh, A., 2014, Yield of tomato as influenced by training and pruning in the Sudan Savanna of Nigeria. Journal of Plant Sciences, 2: 310-317.

Nimje, P. M., Wajnan, O. D. and Shyam, M., 1990, Greenhouse technology for vegetable crop production. Proc. of XI Int. Cong. on the use of Plastics in Agric., pp. 83-90.

Preece, J, E. and Read, P. E., 2005, The biology of horticulture. $2^{\text {nd }}$ Ed: John Wiley and Sons, New York, United States, 1: 528 .

Ranjit, C. and Bandopadhyay, S., 2014, Studies on effect of organic, inorganic and bio-fertilizers on plant nutrient status and availability of major nutrients in tomato. Int. J. Bio-resources and stress mgt., 5(1): 93-97.

Razzak, H. A., Ibrahim, A., Allaha M. W. and Alasadon, A., 2013, Response of cherry tomato (Solanum lycopersicum var. cerasiforme) to pruning system and irrigation rates under greenhouse condition. Asian Journal of Crop Science, 5(1): 1-11.

Septa, N. K., Septa, S. R., Septa, S. and Kumar, A., 2013, Energy use efficiency and cost analysis of tomato under greenhouse and open field production system at Nubra valley of Jammu and Kashmir. Int. J. of Environ Sci., 3(4): 1233-1241.

Sharma, S. K., 1995, Response of boron and calcium nutrition on pant growth, fruit and seed yield of tomato. Veg. Sci., 22: 27-29.

Xin, C. and Jan, C, Y., 2014, Research progress on nitrogen use and plant growth. J. Northeast. Agric. Uni., 21(2): 68-74.

Yadav, S., Ameta, K. D., Sharma, S. K., Dubey, R. B., Rathore, R. S., Kumar, H. and Kapuriya, V. K., 2017, Effect of spacing and training on vegetative 
growth characteristics and yield of tomato (Solanum lycopersicum L.) grown in polyhouse. Int. J. Curr. Microbiol. App. Sci., 6(5): 1969-1976. Yeptho, V., Kanaujia, S. V. B. and Amod, S., 2010, Effect of Integrated Nutrient
Management on growth, yield and quality of tomato under poly house condition. J. Soils and Crops., 22(2): 246-252.

\section{How to cite this article:}

Anand, S.K., N. Basavaraja, C.N. Hanchinamani, H.P. Hadimani, I.B. Biradar and Satish, D. 2018. Influence of Different Training and Nutrition Levels on Growth and Yield of Tomato (Solanum lycopersicum L.) under Protected Condition. Int.J.Curr.Microbiol.App.Sci. 7(09): 3288-3299. doi: https://doi.org/10.20546/ijcmas.2018.709.408 